\title{
Part 1: Possible Graviton Detection, for Outer Space Treatment of the Gertenshehtein Effect. i.e. Dyson's Construction/Analysis Does Not Precludes Earth Bound Generation of Gravitons
}

\author{
Andrew W. Beckwith \\ Physics Department, College of Physics, Chongqing University Huxi Campus, Chongqing, China \\ Email: Rwill9955b@gmail.com, abeckwith@uh.edu
}

How to cite this paper: Beckwith, A.W. (2017) Part 1: Possible Graviton Detection, for Outer Space Treatment of the Gertenshehtein Effect. i.e. Dyson's Construction/ Analysis Does Not Precludes Earth Bound Generation of Gravitons. Journal of High Energy Physics, Gravitation and Cosmolo$g y, 3,1-8$.

http://dx.doi.org/10.4236/jhepgc.2017.31001

Received: October 7, 2016

Accepted: November 13, 2016

Published: November 16, 2016

Copyright $\odot 2017$ by author and Scientific Research Publishing Inc. This work is licensed under the Creative Commons Attribution International License (CC BY 4.0).

http://creativecommons.org/licenses/by/4.0/ c) () Open Access

\begin{abstract}
We are reviewing Freeman Dyson's paper which alleged that detection of gravitons via LIGO, or by outer space experiments (due to probabilistic calculations which we review in the document), an impossibility. The disagreement we have with Dr. Dyson is that his probability calculations are taking place in almost infinite spatial domains, which renders the detection protocols, using his probability scheme, impossible. After we summarize the Dyson outer space arguments, and how Dyson got them, we will refer the reader to the very strain calculation done in the referenced PRD article, so cited, as to how a nuclear weapon could generate GW, and then afterwards, refer the reader to a $2^{\text {nd }}$ paper, of how Tokamaks could detect GW/ Gravitons, as detectable by the 3DSR effect. Nowhere are we suggesting DETONITION of a nuclear device to generate GW! The reader is referred to another Li et al. PRD article, 2008, as to 3DSR, as to how detection of GW/Gravitons could occur due to something other than the Gertenshehtein effect, in this paper, i.e. they can look it up, and then in a $2^{\text {nd }}$ follow up paper learn how a Tokamak could be utilized to have a finite sized geometry, for using the 3DSR effect for GW generation. The first paper highlights how if one assumes that only by use of infinite spatial geometry, and by using only the Gertenshehtein effect, that indeed one can convince oneself as to not bothering with the very real prospects of earthbound generation of Gravitons and GW, and that in doing so, GW research will be strictly limited, even with the outstanding results of LIGO, which in no way should be criticized. The entire analysis makes the case that foundational research as to the nature of GRAVITY means moving beyond the mental limitations place on GW/Graviton research by Dyson's 2009 paper.
\end{abstract}

\section{Keywords}

Gertenshtein Effect, LIGO, Nuclear Detonations 


\section{Introduction}

Our paper is dedicated to two hypothesis. i.e. that if one initially evaluates gravitons as interacting in magnetic fields in an infinite spatial domain, that the supposition given by Dyson, in [1] is, correct. We state that the use of the Gerteshtein effect [2] in outer space does imply that the outer space supposed ban on Graviton detection is definitive, only because of the infinite dimensions. And other issues which revolve about the distance D, and magnetic field Strength B, all of which shows up in our document.

Secondly, we also state that Dyson, in making this construction has by default refused to consider Earth bound generation of Gravitons, and that in doing so, by selection bias, has negated measurement protocol which would not use the Gertenshtein effect, and that all Dyson has done has to shut the door by insisting upon only using Outer space generation of Gravitons, while not considering surface bound generation of Gravitons and Gravity waves. We state this in passing as a bridge to the $2^{\text {nd }}$ paper following this one, which will be to be to elaborate upon what is touched on in [3] as to Tokamaks.

Later, in a $2^{\text {nd }}$ paper after this document we are examining graviton production in a finite sized device cavity, as stated in [3], i.e. a Tokamak, state that there is a different way to analyze the graviton production problem.

Why are we doing this? In a word, Dyson has in [1] mentally referenced extraterrestrial generation of Gravitational waves/gravitons in a configuration which reduces the chance of detection of gravitons to zero.

This selection bias as to GW generation and possible graviton detection due to experimental conditions not involving extraterrestrial generation of GW/Gravitons is shown to be counter intuitive, since as proved in [4] nuclear weapon generation of GW of sufficient magnitude of detection has been mathematically stated as a given.

From [4] we have the following quote:

The possibility of producing gravitational radiation with nuclear explosives rests on the fact that the detonation of an asymmetric distribution of explosive would produce a rapidly varying quadrupole moment. As a hypothetical example, suppose one could arrange to detonate a mixture of deuterium and tritium in a cylindrical shape.

Preliminary investigations with say a .5 megaton blast give a crude strain value of $\mathrm{h} \sim 10^{-28}$ or so. This value of the strain is right in this PRD document.

What the author did, via back of the envelope calculations was to determine that a 15 megaton blast would have instead strain value of $\mathrm{h} \sim 10^{-25}$ to $\mathrm{h} \sim 10^{-26}$.

I.e. in the case of Castle Bravo, 1954, which was a 15 megaton device if one had interferometer based gravitational wave detectors on standby it is not at all inconceivable that gravitational waves could have been detected decades before the 2016 announcement of GW.

The 3DSR measurement machine for GW according to Dr. Li allegedly has a $\mathrm{h} \sim 10^{-25}$ to $\mathrm{h} \sim 10^{-26}$ strain sensitivity. This was given to the author in numerous discussions with Dr. Li, in person, the last time being in December 2015, when the author was shown schematics of the 3DSR as planned by Dr. Li for deployment [5].

A referee objected to referencing [4], thinking that in doing so, that the author, myself, was advocating surface nuclear weapon testing to generate ground based genera- 
tion of GW. This is not relevant. What was intended was to show that GW of a sufficient strain condition could be generated, and this does not mean that the author was advocating violation of the Nuclear test band treaty!

Having said that, it is time to begin the review of the Dyson analysis, and to show its limitations.

\section{Discussion of the Details of the Dyson Analysis, with an Eye toward Its Eventual Inapplicability as to Ruling out GW/Graviton Detection Due to Small Geometry Generation of GW/Gravitons. i.e. the Probability Expression Breaks Down, Completely}

Dyson in [1] derived criteria as to the probability one could obtain physical phenomenon theoretically modeled by the Gertsenshtein effect [2]. The Gertsenshtein effect [2] is the coupling of magnetic fields, gravitons, and photons. In the Dyson treatment [1] of the Gertsenshtein effect [2], Dyson hypothesized distances up to many light years for an interaction of magnetic fields, gravitons and photons, for experimental signals which could be detected on the Earth's surface. This assumed geometry of many light years distance lead to the predicted Gertshenshtein effect [2] unable to allow for graviton detection.

I.e. we emphasize that this only is relevant toward the analysis of extra-terrestrial generation of GW which is ignoring the evidence in [4] which would be for surface bound generation for GW.

Finally, we mention an error in Dyson's argument against LIGO, in which he incorrectly rendered the value of gravitational constant $G$, times 1 solar mass, divided by the speed of light, squared as equal to about $10^{-33}$ centimeters. The correct value is $1.5 \mathrm{ki}-$ lometers.

The upshot, in all of this is that Dyson has mentally ruled out even considering earth bound experiments which could generate GW, and this in spite of [4], which has lead the author to the research suggestion as to Tokamak generation of GW as given in [3] which is elaborated upon in the $2^{\text {nd }}$ follow up paper, to this one, which is the sequel to this document.

\section{Probability for the Gertsentshtein Effect, as Described by Dyson for the GW Thought Experiment for Extraterrestrial Sources}

We will briefly report upon Dyson's well written summary results, passing by necessity to the part on the likelihood of the Gertsenshtein effect occurring in a laboratory environment [1].

In general relativity the metric $g_{a b}(\boldsymbol{x}, t)$ is a set of numbers associated with each point which gives the distance to neighboring points. i.e. general relativity is a classical theory. By necessity, perturbations from flat Euclidian space, are usually configured as ripples in "flat space", which are the imprint of gravitational waves in space-time. Our paper is to first of all give the probability of a pairing of photons to gravitons linkage, the Gertentshtein effect, as to how the signatures of a perturbation to the metric $g_{a b}(\boldsymbol{x}, t)$ is 
linkable to photons and vice versa. The Gertentshtein effect is linked to how there is a linkage, signal wise, between gravitons and photons. To do so let us look at the Dyson criteria as a minimum threshold for the Gertentshtein effect happening [1], namely

$$
D \cdot B^{2} \cdot \omega \leq 10^{43}
$$

The propagation distance is given by $D$, the magnetic field by $B$, and the frequency of gravitational radiation is given by $\omega$. Then by [1] the probability of detection would be

$$
\begin{aligned}
& P=\sin ^{2}(B / L) \sim\left(G B^{2} D^{2} / 4 c^{2}\right) \\
& \& \\
& L=\left(10^{25} / B\right) \\
& \& \\
& P \sim \sin ^{2} \sqrt{\left(10^{36} / B^{2} \cdot \omega^{2}\right)} \propto 10^{36} / B^{2} \cdot \omega^{2} \ll 1
\end{aligned}
$$

Realistically the magnetic field is tiny and reference [2] has that Dyson is only considering going from Photons generated by initial extraterrestrial events to gravitons, due to the details of [2]. In addition the distance D is enormous. Note, that, ironically, Dyson gets further simplification leading to as what he gives in [1] a probability of

$$
P=10^{-24} B^{2}
$$

In the case of astronomical effects, he is effectively precluding ANY detection of gravitons, since the magnetic fields would be tiny. Furthermore the entire effect, above depends upon a photon to graviton conversion effect. Needless to say that in practical details Equation (3) would be less than $10^{-10}$ in value if Dyson's initial assumptions about $\mathrm{D}$ and $\mathrm{B}$ held. However, there is a contradiction which shows up as follows.

The end result of the Dyson analysis is that only if one has ultrahigh frequencies will the last Equation (2) probability vanish. I.e. If one has such a high frequency, as given by Dyson, the of course, Equation (2) P would then be close to zero.

The question is, does Dyson's Equation (2) make sense? Note that all it is assuming is ultrahigh frequencies; i.e. Dyson picked the values of $\mathrm{B}$ and also the picked value of $\omega \sim 10^{20} \mathrm{~Hz}$ is chosen for the purpose of making

$P \sim \sin ^{2} \sqrt{\left(10^{36} / B^{2} \cdot \omega^{2}\right)} \propto 10^{36} / B^{2} \cdot \omega^{2} \ll 1$, i.e. Dyson cherry picked the numbers to make the probability for the Gertsenshtein effect as almost non existent, even if Equation (1) were satisfied.

The question is, why did Dyson pick such an enormous initial frequency $\omega \sim 10^{20} \mathrm{~Hz}$ ? Why the magnetic field magnitude value picked? IMO this looks like cherry picking in order to force the probability of detection of a Graviton to zero.

Secondly, in finite spatial geometry, we would have to go to 3DSR, as Equation (2) would effectively force the probability to zero, since D would be tiny. So, this is why the $2^{\text {nd }}$ paper uses the 3DSR paradigm for evaluation of GW/graviton physics [6] i.e. the ironic supposition is, looking at a literal reading of Equation (2) would be that if the frequency were not ultra high, and D light years in distance, that there would be an approach toward having P probability going to $100 \%$.

Does that last sentence make sense? Seriously. 


\section{Dyson's Analysis of the Earth as a GW Detector}

We now review the particulars of Dyson's analysis of the Earth as a GW detector [1]. In doing so we are using the same numbers, and our break down of the results show that Dyson is making some assumptions here, which need to be seriously reviewed. In debt with the methodology of finding out what is germane in his analysis to research. To begin with, Dysons, Formula (23) as given in Dyson's reference [1] has a next flux of Gravitons hitting the surface of the Earth from the Sun

$$
F \text { (flux) -gravitons hitting Earth }=4 \times 10^{-4} \text { Gravitons per } \mathrm{cm} \text {, squared, per second }
$$

In this formulation of gravitons hitting the surface of the Earth, using Dysons numbers, he claims that only 1 graviton out of 10 to the 32 nd power of gravitons can be detected by the Earth's surface, assuming a graviton has about a kilovolt of energy i.e. this is, in its heart a situation where Dyson [1] is assuming an absorbtion cross section 10 to the minus 41 st power per square centimeter per gram for the Earth, and an absurdly low collision rate. If this were true we are neglecting the Gertsenshtein interaction, since we are assuming no magnetic interface with incoming gravitons. This is only justifiable if there is a hard sphere collision between incoming "gravitons" and ordinary matter.

\section{Looking at the Problem of LIG0, and Reviewing Dyson's Claims}

From the LIGO foundation and the Advanced LIGO PROJECT BOOK [7] there is the following Figure 1.

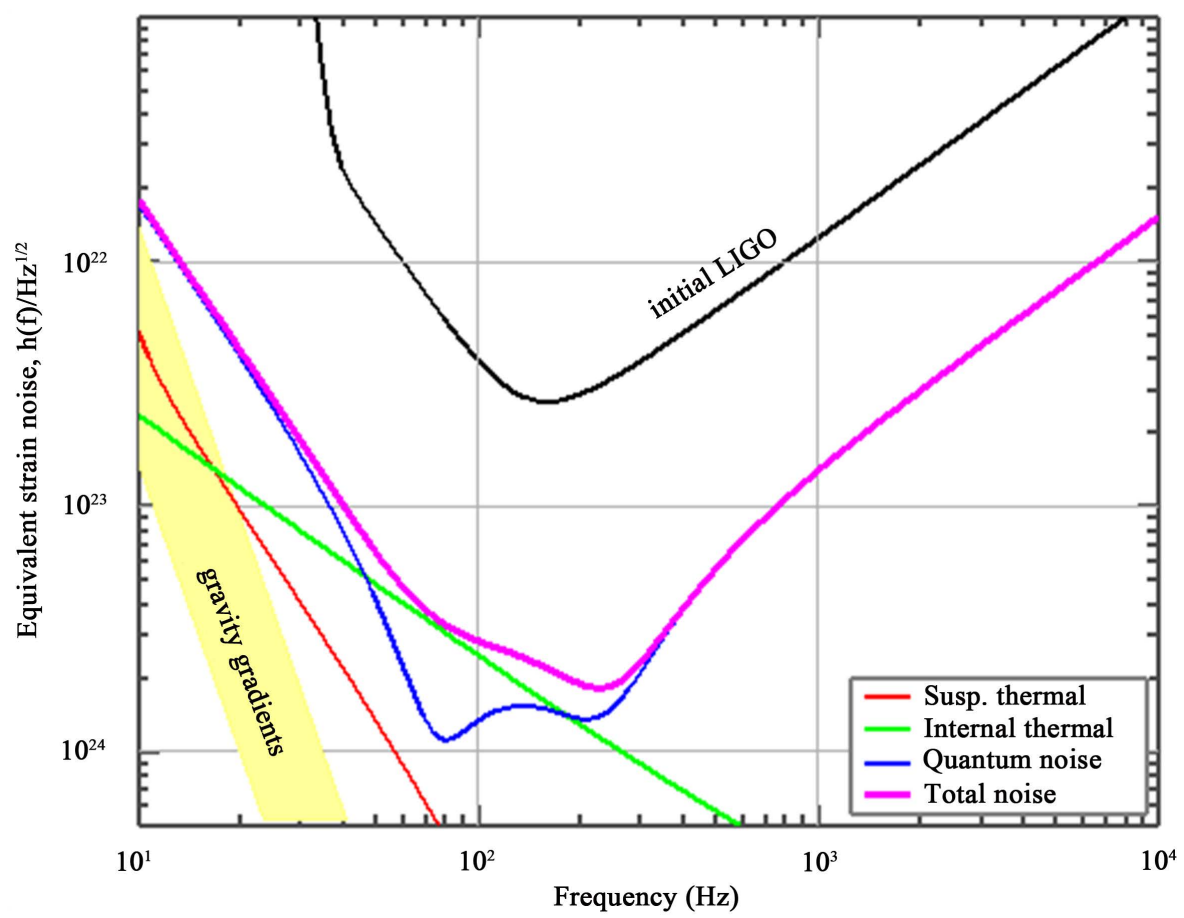

Figure 1. Noise anatomy of advanced LIGO. This model of the noise performance is based on the LIGO current requirements set, and represents the principal contributors of the noise and the least-squares sum of those components expressed as an equivalent gravitational wave strain. Please see reference [7] from the advanced LIGO project book as the source of this Figure 1. 
From the Advanced LIGO PROJECT BOOK in reference [7] comes the following claim, as given in the following quote as given below

Quote: From the Advanced LIGO PROJECT BOOK [7]

- $\mathrm{BH}+\mathrm{BH}$ mergers and ringdowns: When rapidly spinning $\mathrm{BH}$ 's collide, they should trigger large-amplitude, nonlinear oscillations of curved spacetime around their merging horizons. Little is known about the dynamics of spacetime under these extreme circumstances; we can learn about it by comparing LIGO's observations of the emitted waves with supercomputer simulations. Advanced LIGO can detect the merger waves from $\mathrm{BH}$ binaries with total mass as great as 2000 solar mass to cosmological redshifts as large as $\mathrm{z}=2$.

We next will describe the signal strength of the Advanced LIGO device. Once again, note that has amply shown that the signal strength formulation in Equation (5) works superbly.

The signal strength of LIGO as given by LIGO in reference [8] depends upon

$$
h \sim \frac{G M}{c^{2}} \times \frac{1}{r} \times\left[\frac{v}{c}\right]^{2}
$$

Here, $r$ is the distance of this gravitational generation from the detector, and $v / c$ is the ratio of say objects within the gravitational detector, and the speed of light. Usually, $v / c$ is much less than 1. Equation (5) is particularly relevant to the problem of inspiraling black holes falling into each other, and so, now with this, we should review what Dyson had to say about gravitons, and GW, as well as LIGO.

Right before Dyson's section 4 in [1], there is a statement that the frequency rage for a single graviton to kick an electron out of a single atom, which is $10^{15} \mathrm{Hertz}$ [1]. We will later on comment this estimate [1] as a way to obtain a graviton-photon interaction and also refer to Dyson's claim just before his section 5, about thermal graviton generators, that the absorption cross section of ordinary matter (for a graviton) is $10^{-41}$ square centimeters per gram. For LIGO, the frequency range is about $10^{2} \mathrm{~Hz}$ for two black holes inspiraling into each other, not $10^{15} \mathrm{Hertz}$, so the option of having a single graviton displace an electron from an atom, is zero. Which leads us to consider the relation given by Dyson, as his reference [1] has its own Equation (10), namely an upper bound to a minimum separation between two objects, say in a LIGO grid, is given by

$$
\frac{G M}{c^{2}}>D
$$

If $M$ is the mass of the sun, then the L.H.S. of Equation (6) is 1.482 times $10^{3}$ meters, i.e. roughly 1.5 kilometers, or approximately a mile. Assume that then we wish to compare Equation (5) with Equation (6) with a value of $V / c \sim 10^{-3}$, we obtain that two inspiraling black holes with a strain value of $\mathrm{h} \sim 10^{-22}$ are about 1000 light years from Earth, for two black holes, combined mass of about one solar mass.

Note again, that reference [9] shows that LIGO works quite well, so there is no reason to doubt Equation (5)!

\section{Conclusion, Looking at the Problem of Mass of a Graviton, and the Nature of GW}

Ultimately the following would have to be investigated, if we wish to know if Gravitons 


$$
\begin{aligned}
& \Theta\left(t-t_{\text {Planck }}\right)=0, t<t_{\text {Planck }} \\
& \Theta\left(t-t_{\text {Planck }}\right)=1, t>t_{\text {Planck }} \\
& m_{g}=0 ; t<t_{\text {Planck }} \\
& m_{\text {graviton }}=\frac{\hbar}{c} \cdot \sqrt{\frac{\left(2 \Lambda_{\text {today }}\right)}{3}} \approx \sqrt{\frac{(2 \Lambda)}{3}} ; t>t_{\text {Planck }} \\
& m_{\text {graviton }}=\frac{\hbar}{c} \cdot \sqrt{\frac{\left(2 \cdot \Theta\left(t-t_{\text {Planck }}\right) \cdot \Lambda_{\text {today }}\right)}{3}}
\end{aligned}
$$

have mass, and how this would affect measurement of gravitons [10].

Equation (7) defines the range of values for graviton mass which need to be considered. i.e. the interesting point is that Pre-Planckian gravitons would be massless whereas the later day gravitons would have a small mass, and the point of transition between the two forms of graviton mass would likely influence the relic conditions which would show up in choosing between the alternatives given in [11] as to the nature of gravity itself [9].

This would be a great refinement if verified in the determination of the foundations of gravity, and also is integral to the 3DSR inquiry which would show up in the $2^{\text {nd }}$ paper, as to GW and Gravitons which involves Tokamaks.

We also leave open, the possibility of massive gravitons as given by [12] which would presumably have observational consequences which may show up; in CMBR readings, and other places, once GW astronomy becomes a fully vetted and understood experimental scientific discipline.

Having said that, we urge the readers to review the 3DSR Tokamak document next.

\section{Acknowledgements}

This work is supported in part by National Nature Science Foundation of China grant No. 11375279.

The present document is presented to JHEPGC, on account of the refusal of Hindawi, in particular Advances in High energy physics to heed the recommendation of two invited Editors, for inclusion of this document in a special issue of Quantum gravity and applications. Contrary to the wishes of the two invited editors for the special edition, Advances in High energy physics authorized. Due to a violation of protocol, by Advances in high energy physics, this document and its companion piece was pulled from Advances in high energy physics and re submitted to the JHEPGC journal. This is due to Advances in high energy physics refusing to follow journalistic review protocol and micromanaging the acceptance of this document when in fact it was deemed worthy of acceptance by the invited editors.

\section{Conflict of Interest}

Statement as to there being no conflict of interest: The author declares that there is no conflict of interest as to the publication of this document to the best of his knowledge.

Statement being that there is no conflict of interest due to the support of the National Nature foundation of China for the publishing of this document. 


\section{References}

[1] Dyson, F. (2013) Is a Graviton Detectable? International Journal of Modern Physics A, 28, Article ID: 1330041 (14 p).

https://publications.ias.edu/sites/default/files/poincare2012.pdf

[2] Gertsenshtein, M.E. (1961) Wave Resonance of Light and Gravitational Waves. Journal of Experimental and Theoretical Physics, 41, 113-114, English Translation in Soviet Physics JETP, 14, 84-85 (1962).

[3] Beckwith, A. Review of the Grischuk and Sachin Gravitational Wave Generator Via Tokamak Physics. http://vixra.org/abs/1311.0132

[4] Chapline, G.F., Nuckolls, J. and Wood, L.L. (1974) Gravitational-Radiation Production Using Nuclear Explosions. Physical Review D, 10, 1064-1065.

https:/doi.org/10.1103/PhysRevD.10.1064

[5] Private Discussion with Dr. Li Fangyu, November 15, 2015, in Chongqing University (Shown the 3DSR Technology Schematics Used for a Presentation in Beijing, by Dr. Li).

[6] Li, F.Y., Yang, N., Fang, Z.Y., Baker Jr., R.M.L., Stephenson, G.V. and Wen, H. (2009) Signal Photon Flux and Background Noise in a Coupling Electromagnetic Detecting System for High Frequency Gravitational Waves. Physical Review D, 80, Article ID: 064013. http://arxiv.org/abs/0909.4118

[7] Advanced LIGO PROJECT BOOK. https://labcit.ligo.caltech.edu/ dhs/Adv-LIGO/old/main-p.pdf

[8] http://www.tapir.caltech.edu/ teviet/Waves/gwave.html

[9] Abbott, B.P., et al., LIGO Scientific Collaboration and Virgo Collaboration (2016) Observation Gravitational Waves from a Binary Black Hole Merger. Physical Review Letters, 116, Article ID: Article ID: 061102. https://physics.aps.org/featured-article-pdf/10.1103/PhysRevLett.116.061102

[10] Haranas, I. and Gkigkitzis, I. (2014) The Mass of Graviton and Its Relation to the Number of Information According to the Holographic Principle. International Scholarly Research Notices, 2014, Article ID: 718251. https:/doi.org/10.1155/2014/718251

[11] Corda, C. (2009) Interferometric Detection of Gravitational Waves: The Definitive Test for General Relativity. International Journal of Modern Physics D, 18, 2275-2282.

http://arxiv.org/abs/0905.2502 https:/doi.org/10.1142/S0218271809015904

[12] Sepehri, A. and Ali, A.F. Birth and Growth of Nonlinear Massive Gravity and It's Transition to Nonlinear Electrodynamics in a System of Mp-Branes.

http://arxiv.org/pdf/1602.06210.pdf 
Submit or recommend next manuscript to SCIRP and we will provide best service for you:

Accepting pre-submission inquiries through Email, Facebook, LinkedIn, Twitter, etc. A wide selection of journals (inclusive of 9 subjects, more than 200 journals) Providing 24-hour high-quality service

User-friendly online submission system

Fair and swift peer-review system

Efficient typesetting and proofreading procedure

Display of the result of downloads and visits, as well as the number of cited articles Maximum dissemination of your research work

Submit your manuscript at: http://papersubmission.scirp.org/

Or contact jhepgc@scirp.org 